\title{
Differential expression of lamin B1 in triple negative breast cancer.
}

Shahan Mamoor, MS ${ }^{1}$

$3 \quad$ shahanmamoor@gmail.com

East Islip, NY 11730

Women diagnosed with triple negative breast cancer can benefit neither from endocrine therapy nor from HER2-targeted therapies ${ }^{1}$. We mined published microarray datasets ${ }^{2,3}$ to determine in an unbiased fashion and at the systems level genes most differentially expressed in the primary tumors of patients with breast cancer. We report here significant differential expression of the gene encoding lamin B1, LMNB1, when comparing the tumor cells of patients with triple negative breast cancer to normal mammary ductal cells ${ }^{2}$. LMNB1 was also differentially expressed in bulk tumor in human breast cancer ${ }^{3}$. LMNB1 mRNA was present at significantly increased quantities in TNBC tumor cells relative to normal mammary ductal cells. Analysis of human survival data revealed that expression of LMNB1 in primary tumors of the breast was correlated with overall survival in patients with basal-like and luminal A subtype cancer, while within triple negative breast cancer, primary tumor expression of LMNB1 was correlated with overall survival in patients with basal-like 2, immunomodulatory and mesenchymal subtype disease. LMNB1 may be of relevance to initiation, maintenance or progression of triple negative breast cancers.

Keywords: breast cancer, LMNB1, lamin B1, systems biology of breast cancer, targeted therapeutics in breast cancer. 
Patients with hormone receptor positive disease can benefit from endocrine therapy ${ }^{4}$ : aromatase inhibition such as that provided by exemestane or letrozole, or estrogen receptor antagonism such as that provided by tamoxifen or fulvestrant. Patients whose tumors overexpress the human epidermal growth factor 2 (HER2) can be treated with HER2-targeted therapies: HER2 inhibitors, immunoglobulin ${ }^{5}$ - or small molecule-based ${ }^{6}$. As triple negative breast cancer (TNBC) is defined by its lack of HER2 expression as well as by its lack of expression of the hormone receptor estrogen, patients diagnosed with triple negative breast cancer will not benefit from either of these treatment strategies.

Understanding the transcriptional composition of triple negative breast cancers in humans can facilitate development of novel therapeutics and contribute to efforts to prevent progression to metastatic stages. We mined published microarray data ${ }^{2,3}$ to understand at the transcriptome level and in an unbiased fashion genes most differentially expressed in the tumor cells of patients with triple negative breast cancer.

\section{Methods}

We utilized datasets GSE38959 ${ }^{2}$ and GSE109169³ for this differential gene expression analysis of female breast cancer in conjunction with GEO2R. GSE38959 was generated using Agilent-014850 Whole Human Genome Microarray 4x44K G4112F technology with $n=13$ samples of normal mammary gland ductal cells and $n=30$ samples of tumor cells from patients with triple negative breast cancer; analysis was performed using platform GPL4133. GSE109169 was generated using Affymetrix Human Exon 1.0 ST Array technology with $n=25$ normal breast tissue and $n=25$ tumors of the breast; analysis was performed using platform GPL5175. The tissues from this dataset are paired tissues ( 25 tumors matching 25 normal breast tissues from 25 patients). The Benjamini and Hochberg method of $p$-value adjustment was used for ranking of differential expression but raw $p$-values were used to assess statistical significance of global differential expression. Log-transformation of data was auto-detected, and the NCBI-generated category of platform annotation was used. A statistical test was performed to evaluate whether LMNB1 expression was significantly different between primary breast tumors and breast tissue using a two-tailed t-test.

For Kaplan-Meier survival analysis, we used the Kaplan-Meier plotter tool ${ }^{7}$ for correlation of LMNB1 mRNA expression levels with overall survival (OS) in $n=431$ patients with basal subtype cancer, $n=596$ patients with luminal A subtype cancer, $n=439$ patients with luminal B subtype cancer, $n=362$ patients with HER $2+$ cancer, and $n=51$ patients with normal-like subtype cancer. In patients with triple negative breast cancer $^{7}$, we analyzed overall survival (OS) for $n=103$ patients with basal-like 1 subtype TNBC, $n=58$ patients with basal-like 2 subtype TNBC, $n=149$ patients with immunomodulatory subtype TNBC, $n=116$ patients with luminal androgen receptor subtype TNBC, $n=114$ patients with mesenchymal subtype TNBC, and $n=39$ patients with mesenchymal stem-like subtype TNBC.

\section{Results}

We performed discovery of genes associated with triple negative breast cancer in humans by mining two independently published microarray datasets ${ }^{2,3}$.

\section{LMNB1 is differentially expressed in primary tumors and tumor cells of the breast.}

Comparison of the tumor cells of patients with triple negative breast cancer to normal mammary ductal cells ${ }^{2}$ revealed lamin B1, encoded by LMNB1, as among the genes whose expression was most significantly different in human TNBC as compared to the normal breast (Chart 1). When sorting each of the genes expressed in the tumor cells of patients with triple negative breast cancer based on significance of difference as compared to normal mammary ductal cells, LMNB1 ranked 233 out of 45015 total transcripts, equating to $99.5 \%$ differential expression (Chart 1). Differential expression of LMNB1 in the tumor cells of patients with triple negative breast cancer was statistically significant (Chart 1; $p=5.56 \mathrm{E}-11)$. 
Analysis of a second microarray datase ${ }^{3}$, here comparing 25 normal breast tissues to 25 tumors of the breast revealed that LMNB1 was also among the genes most differentially expressed in human breast cancer, here in the tumors of women diagnosed with early-onset breast cancer (Chart 2). When sorting each of the genes expressed in tumors of the breast based on significance of difference as compared to normal breast tissue, LMNB1 ranked 1073 out of 19076 total transcripts, equating to $94.4 \%$ differential expression (Chart 2). Differential expression of LMNB1 in female breast cancer was statistically significant (Chart 2; $p=9.70 \mathrm{E}-09)$.

These data indicated that differential expression of LMNB1 was not an artifact of a single microarray dataset, was not an artifact of laser microdissection, and suggested that perturbed expression of LMNB1 was likely a transcriptional feature of triple negative breast cancers and early-onset human breast cancers.

\section{LMNB1 is expressed at significantly higher levels in triple negative tumor cells as compared to} normal cells of the breast.

We obtained exact mRNA expression levels for LMNB1 - from normal mammary ductal cells of the breast and from tumor cells of patients with triple negative breast cancer - to understand the magnitude and direction of LMNB1 expression change in TNBC. LMNB1 mRNA transcript was present at higher levels in triple negative tumor cells of the breast as compared to normal mammary ductal cells (Figure 1). Increased expression of LMNB1 in primary tumor-isolated triple negative breast cancer cells was statistically significant (Figure 1: $p<0.0001$ ). LMNB1 was expressed at $59.7 \pm 83.2$ arbitrary units (A.U.) in normal mammary ductal cells, while it was expressed at $542.9 \pm 344.2$ A.U. in tumor cells of patients with triple negative breast cancer. We calculated a mean fold change of 9.09 when comparing triple negative breast cancer cells to normal mammary ductal cells.

\section{LMNB1 expression correlates with survival outcomes in basal-like and luminal A human breast} cancer.

We performed Kaplan-Meier survival analysis to evaluate potential relationships between primary tumor LMNB1 mRNA expression levels and survival outcomes in patients with breast cancer, when analyzing outcomes based on the molecular subtype of the patient (PAM50 classification): luminal A, luminal B, HER2+, normal-like, and basal-like. We observed a statistically significant correlation between LMNB1 expression and overall survival (OS) in patients with basal-like subtype breast cancer (Figure 2; $\log$ rank $p$-value: 0.016 for overall survival, hazard ratio: 0.63 (0.42-0.92) (Fig. 2)). LMNB1 mRNA levels were a positive prognostic indicator in basal-like breast cancer patients. OS was, on average, markedly shorter for basal-like patients with low tumor expression of LMNB1 as compared to basal-like patients with high tumor expression of LMNB1 (Chart 3).

We also observed a statistically significant correlation between LMNB1 expression and overall survival (OS) in patients with luminal A subtype breast cancer (Figure 2; log rank $p$-value: 0.016 for overall survival, hazard ratio: $1.71(1,1-2,66)$ (Fig. 2)). LMNB1 mRNA levels were a negative prognostic indicator in luminal A breast cancer patients. Median OS was 179.01 months for luminal A patients with low tumor expression of LMNB1 while median OS was 175.46 months for luminal A patients with high tumor expression of LMNB1 (Chart 3).

LMNB1 primary tumor expression was not correlated with overall survival in luminal B subtype (Figure 2; log rank $p$-value: 0.71 for OS, hazard ratio: 0.93 (0.66-1.33) (Fig. 2)), in HER2+ breast cancer (Figure 2; log rank $p$-value: 0.47 for OS, hazard ratio: 1.15 (0.78-1.7 (Fig. 2)), or in the normal-like subtype (Figure 2; log rank $p$-value: 0.64 for OS, hazard ratio: 0.79 (0.29-2.13) (Fig. 2)). 
Influence of primary tumor LMNB1 expression on survival outcomes based on TNBC molecular subtype.

We also performed Kaplan-Meier survival analysis to examine relationships between LMNB1 mRNA expression levels with survival strictly in patients with triple negative breast cancer, analyzing outcomes based on the TNBC molecular subtype ${ }^{8}$ of the patient: basal-like 1, basal-like 2, immunomodulatory, mesenchymal, mesenchymal stem-like, and luminal androgen receptor. We observed a statistically significant correlation between LMNB1 expression and overall survival (OS) in TNBC patients with basal-like 2 subtype disease (Figure 3; log rank $p$-value: 0.0083 for overall survival, hazard ratio: 4.28 (1.33-13.77) (Fig. 3)). LMNB1 mRNA levels were a negative prognostic indicator in patients with basal-like 2 TNBC. OS was, on average, markedly greater for basal-like 2 TNBC patients with low tumor expression of LMNB1 as compared to basal-like 2 TNBC patients with high tumor expression of LMNB1 (Chart 4).

We also observed a statistically significant correlation between LMNB1 expression and overall survival (OS) in TNBC patients with immunomodulatory subtype disease (Figure 3; log rank $p$-value: 0.0059 for overall survival, hazard ratio: 0.31 (0.13-0.75) (Fig. 3)). LMNB1 mRNA levels were a positive prognostic indicator in patients with immunomodulatory TNBC. OS was, on average, markedly shorter for immunomodulatory TNBC patients with low tumor expression of LMNB1 as compared to immunomodulatory TNBC patients with high tumor expression of LMNB1 (Chart 4).

We also observed a statistically significant correlation between LMNB1 expression and overall survival (OS) in TNBC patients with mesenchymal subtype disease (Figure 3; log rank $p$-value: 0.025 for overall survival, hazard ratio: 0.47 (0.24-0.92) (Fig. 3)). LMNB1 mRNA levels were a positive prognostic indicator in patients with mesenchymal TNBC. Median OS was 30.42 months for mesenchymal TNBC patients with low tumor expression of LMNB1 while median OS was 85.2 months for mesenchymal TNBC patients with high tumor expression of LMNB1 (Chart 4).

LMNB1 primary tumor expression was not correlated with overall survival in basal-like 1 subtype (Figure 3; log rank $p$-value: 0.44 for OS, hazard ratio: 0.74 (0.34-1.6) (Fig. 3), in the mesenchymal stem-like subtype (Figure 3; log rank $p$-value: 0.84 for OS, hazard ratio: $1.11(0.4-3.06)$ (Fig. 3)) or in the luminal androgen receptor subtype (Figure 3; log rank $p$-value: 0.49 for OS, hazard ratio: $0.81(0.44-1.48)$ (Fig. 3)).

\section{Discussion}

We performed comparative transcriptome analysis using two independently published microarray datasets $^{2,3}$ to define and catalog transcriptional differences in human triple negative breast cancer. We provided evidence here that differential expression of lamin B1, encoded by the LMNB1 gene, is a defining transcriptional feature of triple negative breast cancer. LMNB1 was also differentially expressed in the bulk tumors of patients with early-onset breast cancer. Expression of LMNB1 was correlated with overall survival in patients with basal-like and luminal A subtype breast cancer, and within triple negative breast cancer patients, primary tumor expression of LMNB1 was correlated with overall survival in patients with basal-like 2, immunomodulatory and mesenchymal subtype disease. Lamin B1 and the molecular processes to which it pertains to may be relevant to the initiation or progression of triple negative breast cancer in humans. 


\section{References}

1. Foulkes, W.D., Smith, I.E. and Reis-Filho, J.S., 2010. Triple-negative breast cancer. New England journal of medicine, 363(20), pp.1938-1948.

2. Komatsu, M., Yoshimaru, T., Matsuo, T., Kiyotani, K., Miyoshi, Y., Tanahashi, T., Rokutan, K., Yamaguchi, R., Saito, A., Imoto, S. and Miyano, S., 2013. Molecular features of triple negative breast cancer cells by genome-wide gene expression profiling analysis. International journal of oncology, 42(2), pp.478-506.

3. Chang, J.W., Kuo, W.H., Lin, C.M., Chen, W.L., Chan, S.H., Chiu, M.F., Chang, I.S., Jiang, S.S., Tsai, F.Y., Chen, C.H. and Huang, P.H., 2018. Wild-type p53 upregulates an early onset breast cancer-associated

4. Spring, L.M., Gupta, A., Reynolds, K.L., Gadd, M.A., Ellisen, L.W., Isakoff, S.J., Moy, B. and Bardia, gene GAS7 to suppress metastasis via GAS7-CYFIP1- mediated signaling pathway. Oncogene, 37(30), pp.4137-4150. and meta-analysis. JAMA oncology, 2(11), pp.1477-1486.

5. Slamon, D., Eiermann, W., Robert, N., Pienkowski, T., Martin, M., Press, M., Mackey, J., Glaspy, J., Chan, A., Pawlicki, M. and Pinter, T., 2011. Adjuvant trastuzumab in HER2-positive breast cancer. New England journal of medicine, 365(14), pp.1273-1283.

6. Park, J.W., Liu, M.C., Yee, D., Yau, C., van’t Veer, L.J., Symmans, W.F., Paoloni, M., Perlmutter, J., Hylton, N.M., Hogarth, M. and DeMichele, A., 2016. Adaptive randomization of neratinib in early breast cancer. New England Journal of Medicine, 375(1), pp.11-22.

7. Györffy, B., Lanczky, A., Eklund, A.C., Denkert, C., Budczies, J., Li, Q. and Szallasi, Z., 2010. An online survival analysis tool to rapidly assess the effect of 22,277 genes on breast cancer prognosis using microarray data of 1,809 patients. Breast cancer research and treatment, 123(3), pp.725-731.

8. Lehmann, B.D., Bauer, J.A., Chen, X., Sanders, M.E., Chakravarthy, A.B., Shyr, Y. and Pietenpol, J.A., 2011. Identification of human triple-negative breast cancer subtypes and preclinical models for selection of targeted therapies. The Journal of clinical investigation, 121(7), pp.2750-2767. 


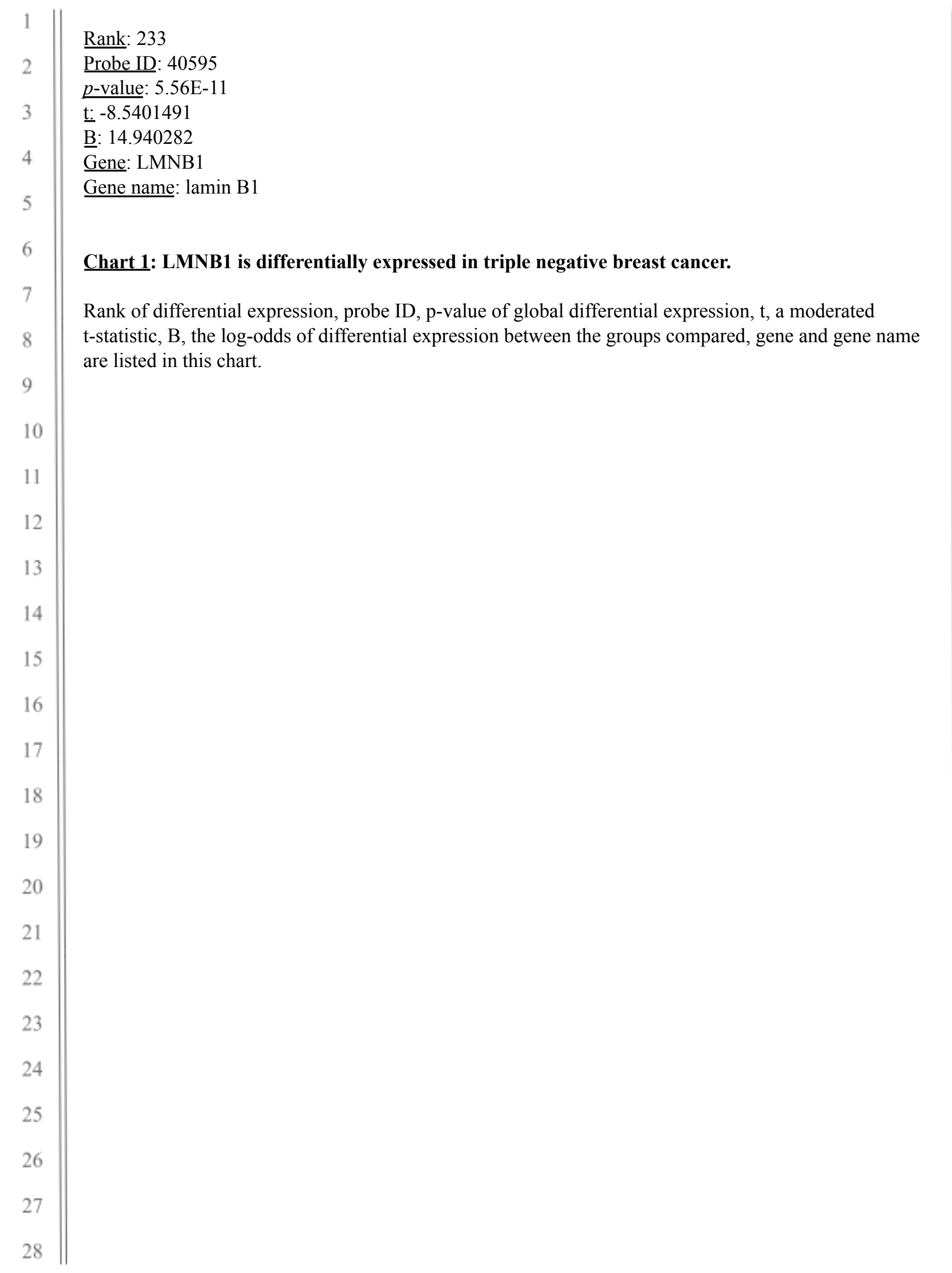




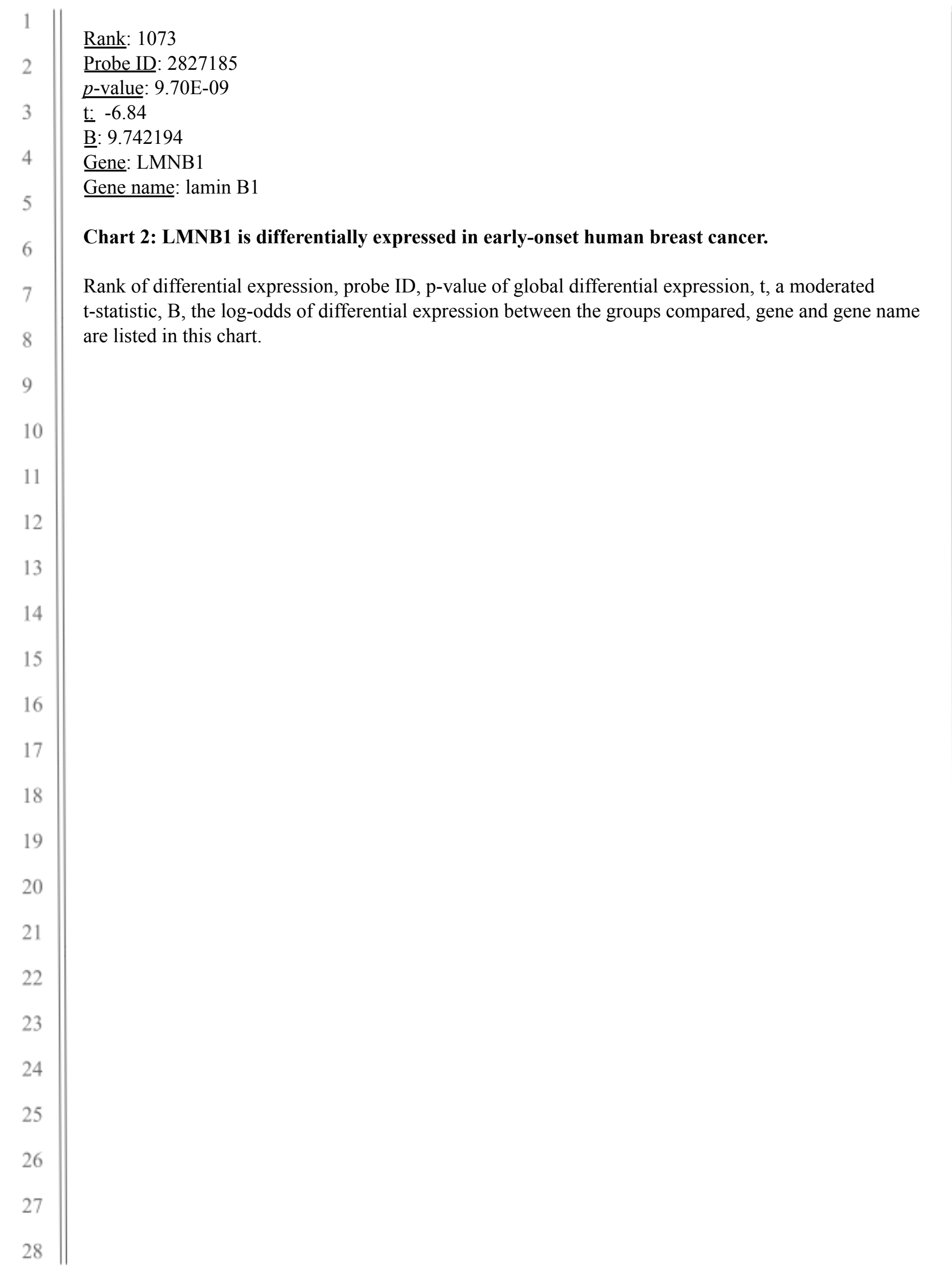




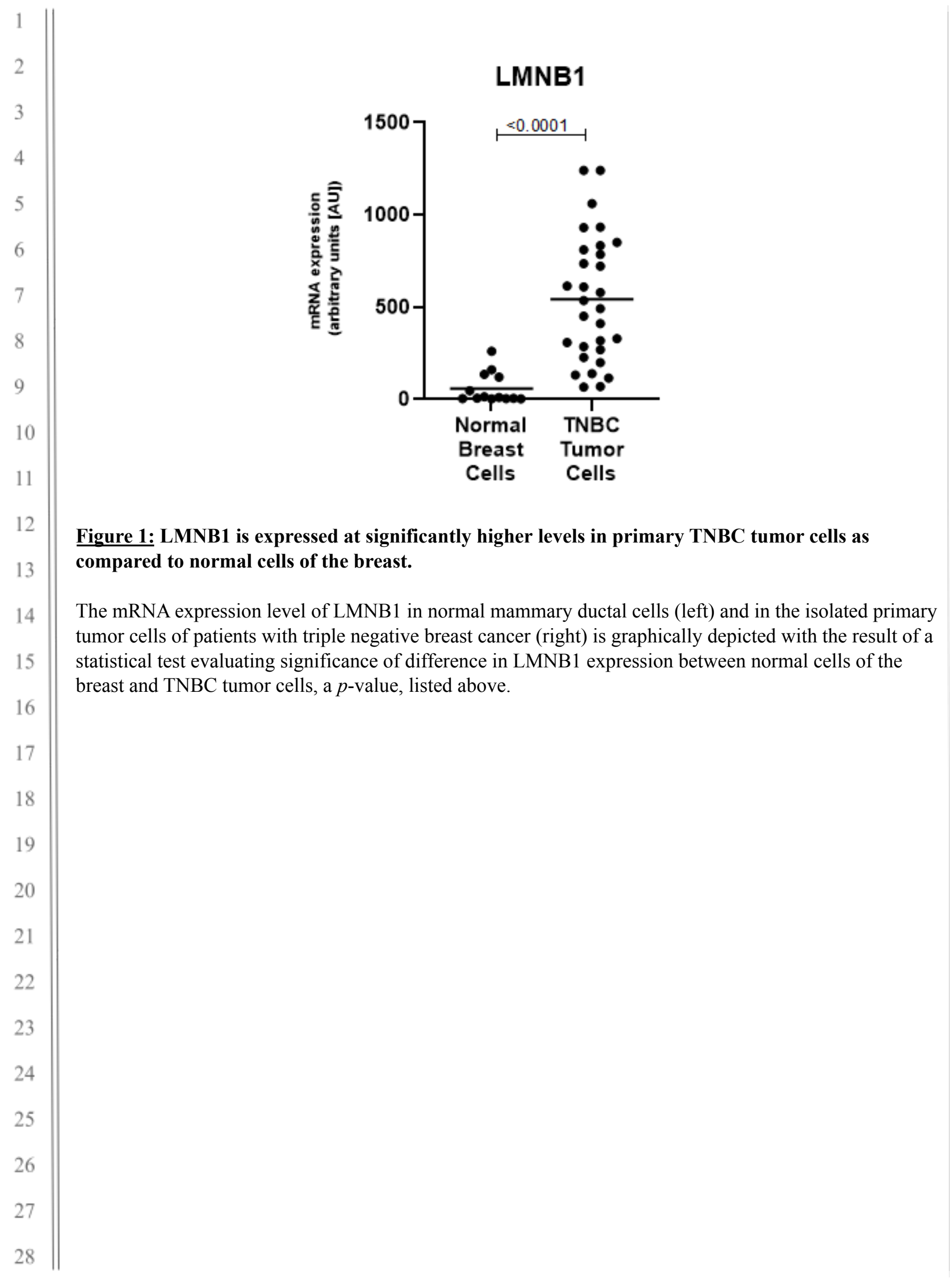




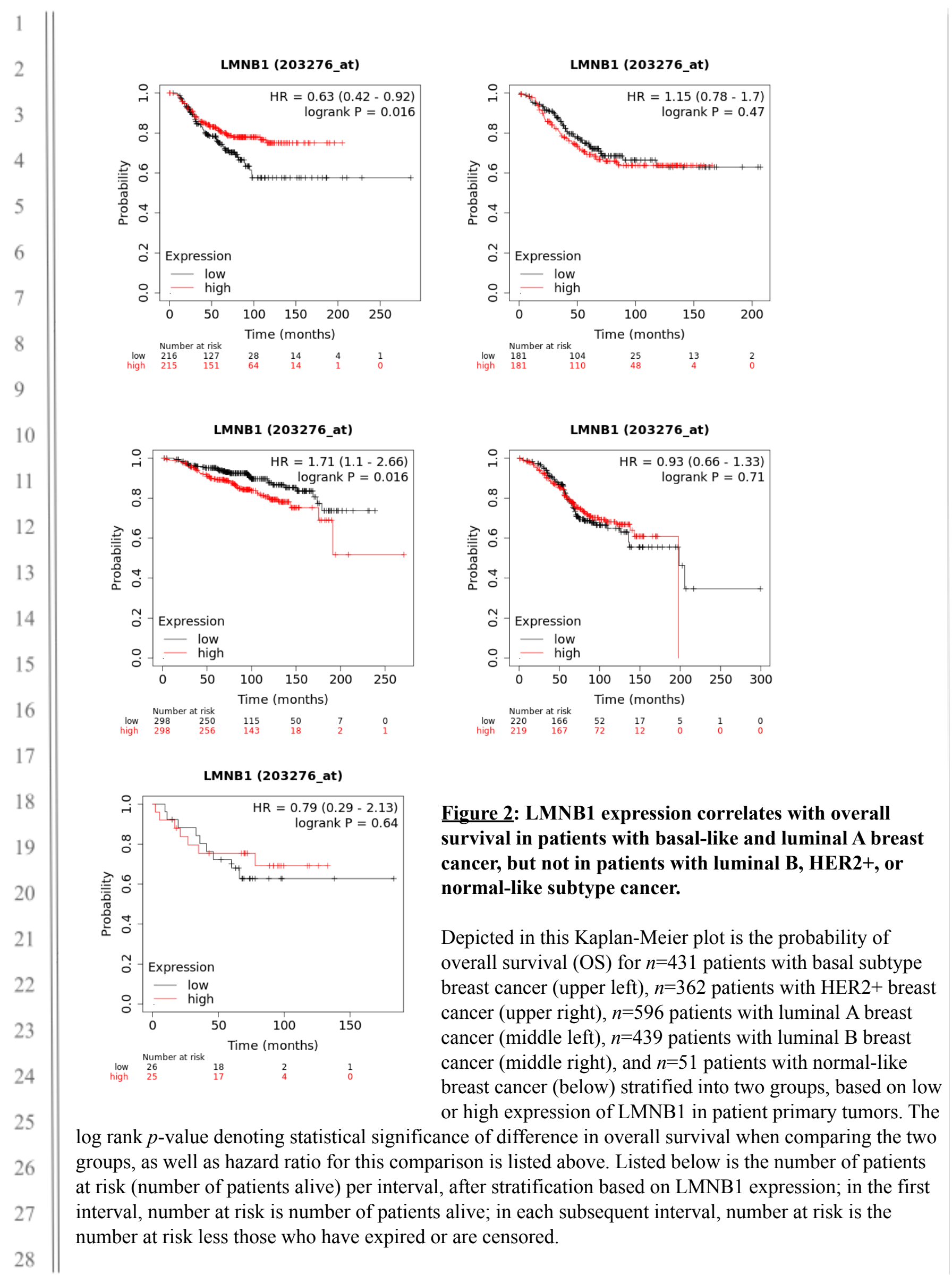




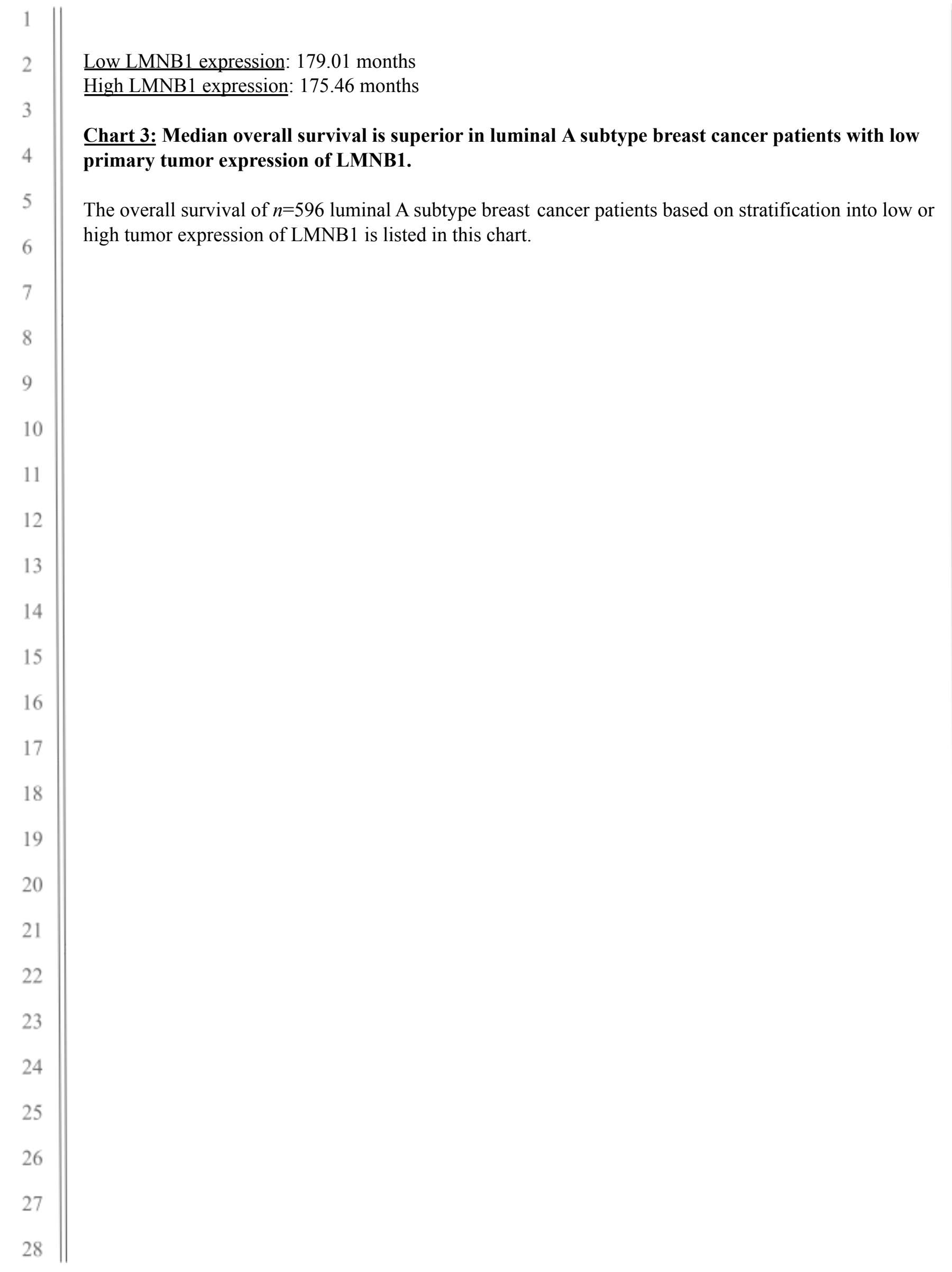




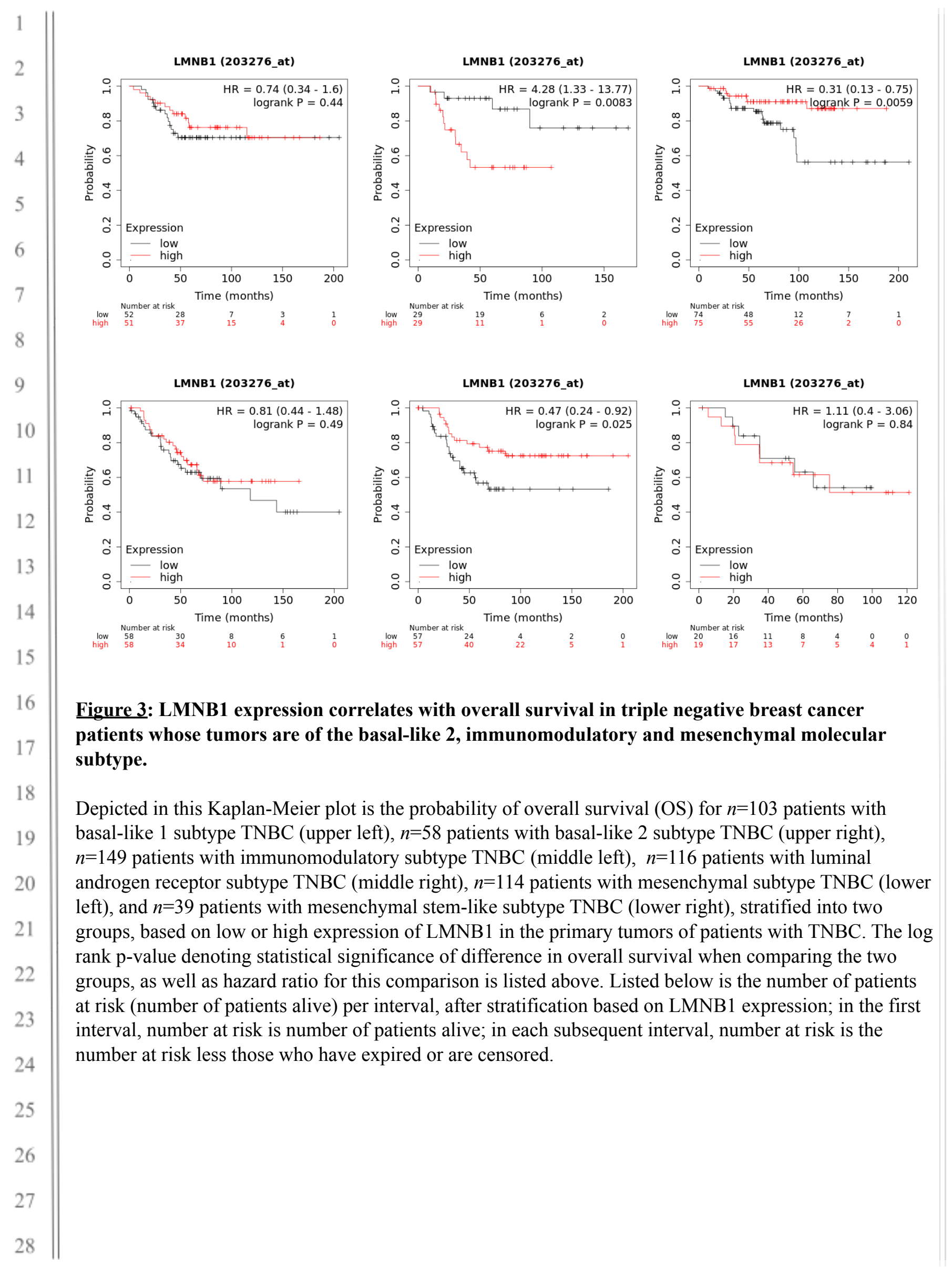




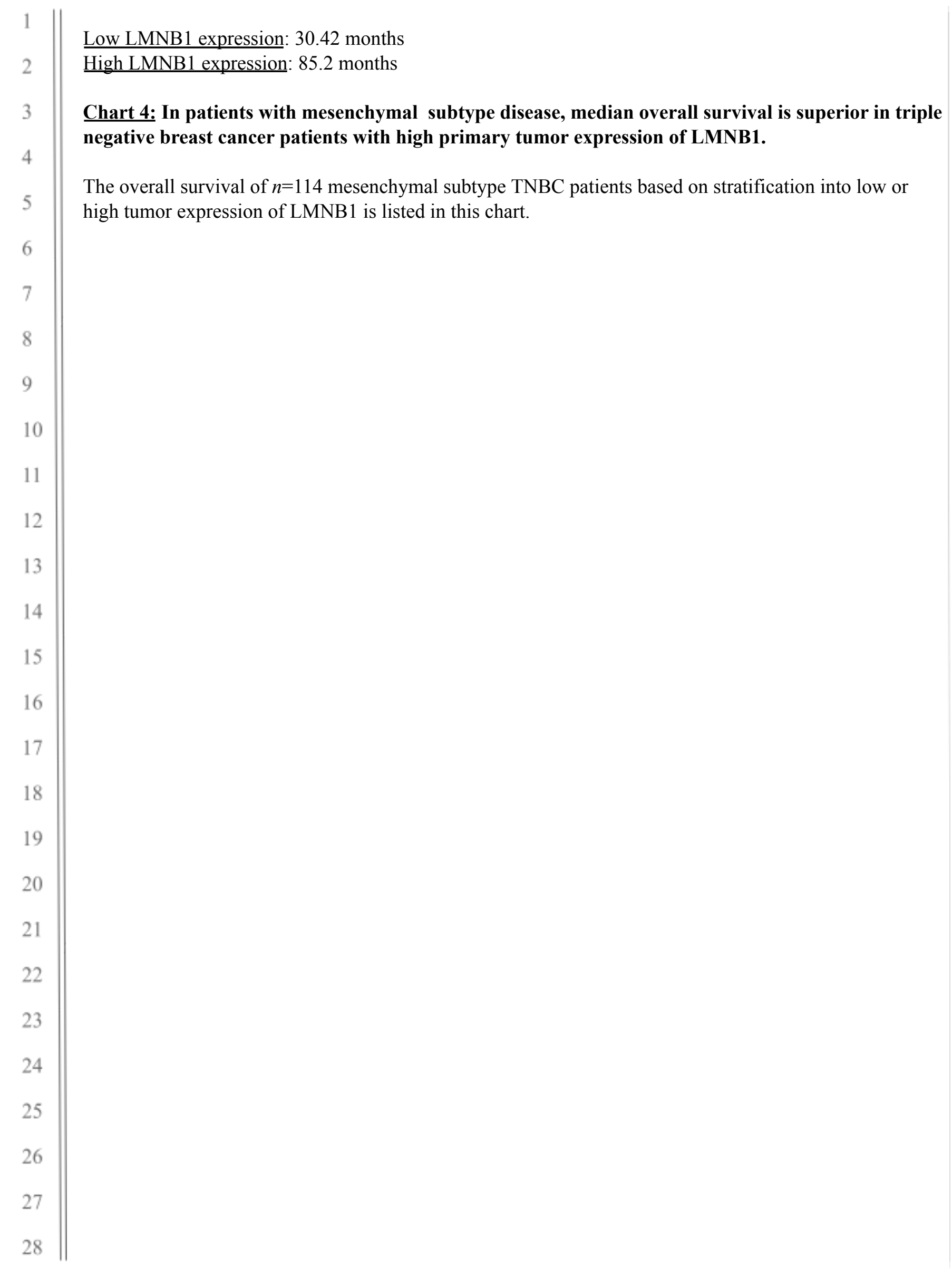

